\title{
A NEURO-FUZZY CLASSIFICATION SYSTEM FOR PATTERN RECOGNITION OF CONTROL CHARTS
}

\author{
Hindi A. Al-Hindi \\ Associate Professor, Department of Quantitative \\ Methods, College of Business and Economics, \\ King Saud University, Al-Qasseem \\ Branch, Al-Melaida, P.O.Box 6033.
}

\begin{abstract}
In the past years, neuro-fuzzy systems received an increasing attention and were used to solve a wide range of problems in different domains. A neuro-fuzzy system is a hybrid system consisting of an artificial neural network and a fuzzy inference system where the learning algorithm of the artificial neural network is used to adjust the parameters of the membership functions associated with the fuzzy inference system. This paper proposes a neuro-fuzzy classification approach for identifying control chart patterns in order to uncover the behavior of the production process. The proposed approach was implemented by building a neuro-fuzzy classification system and by using simulated data. Numerical results showed that the proposed approach has a good recognition performance of patterns on control charts.
\end{abstract}

KEY WORDS: Neuro-Fuzzy System, Control Chart, Pattern Recognition

\section{INTRODUCTION}

Manufacturing organizations are continuously striving to improve the quality of their products through applying more advanced techniques and methods. Technological advances have resulted in a number of artificially intelligent systems that enable the organization to efficiently process the data collected from the production process and induce valuable information. Such information is necessary for management to control the production process. Production process control is the keystone for the success: of the manufacturing organization to ensure that its products are produced with a high level of quality [1].|2\}.

Process control is aimed at reducing deviations in the process to the lowest level possible. Reduced deviations make the process more manageable

\footnotetext{
Manuscript received from Dr. Hindi A. Al- Hindi

Accepted on : $19 / 5 / 2001$

Engineering Research Journal Vol 24,No 3; 2001 Minufiya University, Faculty of Engineering , Shebien El-Kom, Egypt, ISSN 1110-1180
} 
with process outputs closer to the desired values [3]. It is the responsibility of management to reduce deviations through process improvement, investing in new technology and/or applying more advanced techniques to manage the process [2],[4]. Control charts are major tools for process control and improvement in the short term. In addition, they can be used to uncover the behavior of the process in the long run. This can be achieved by applying an efficient pattern recognition technique to identify different patterns present on the control charts.

Neuro-fuzzy systems are receiving an increasing attention in recent years for solving a wide range of problems. A neuro-fuzzy system is a combination of artificial neural network and a fuzzy inference system in such a way that the neural network learning algorithm is used to adjust parameters of the fuzzy sets associated with the fuzzy inference system [5],[6]. In this case, the performance of the neuro-fuzzy system is improved by using the neural network learning methods. In addition, the system can be represented in terms of fuzzy IF_THEN rules capable of handling ambiguous knowledge encountered in real life situations. Examples of such neuro-fuzzy systems include the adaptive network based fuzzy inference system (ANFIS) [7], neuro-fuzzy control (NEFCON) [8] and neuro-fuzzy classification (NEFCLASS) [9].

The goal of this paper is to present an efficient approach for identifying unusual patterns on control charts based on the neuro-fuzzy classification. This approach is intended to uncover unusual behavior of the production process and enhance the utilization of control charts by applying the neuroluzzy classification approach. This provides the ability to detect unusual behavior of the process and use information on the chart more efficiently in controlling the production process. The rest of the paper is organized as follows. Section 2 gives brief description of control charts. Section 3 reviews some of the techniques used for pattern recognition of control charts. Section 4 presents the neuro-fuzzy classification. Section 5 describes the approach used to generate the patterns in order to train and test the neuro-fuzzy system. Section 6 provides the training results of the proposed approach. Section 7 concludes this paper.

\section{QUALITY CONTROL CHARTS}

Statistical process control (SPC) is an approach intended to cnsure that the production process is kept under control [3]. [4]. It utilizes control charts to detect any unusual variations in the process in order for management to take corrective actions to ensure the quality of the products. Process variation which is the central issue of statistical process control can be divided into two components: natural and special variations. Natural process variations are the natural occurring Muctuations inherent in the process. Special cause variations are rypically caused by some problem in the production system that should be eliminated [2].

Control charts are widely used to analyze data collected from a production process and predict the behavior of the process in the future. In addition. control charts cnable management to determine when the process is 
out of control. This is the case when a special cause variation present because something unusual is occurring in the production process. The process is then investigated to uncover the cause of the out of control condition and take appropriate actions.

Control charts can be classified into two types: measurement charts and attribute charts depending on the type of quality characteristic that they are used to monitor [2],[4]. Measurement charts include charts which track sample mean ( $\mathrm{x}$-bar charts) and sample range ( $\mathrm{R}$ charts). For controlling quality characteristic that represents an attribute of the product, $\mathrm{p}$-chart and $\mathrm{c}$ chart are commonly used. The data collected from the production process are used to construct the control chart and if the process is found to be out of control, corrective actions have to be taken to remove assignable causes of the deviations.

$A$ control chart has a centerline corresponding to the average value of the observations and two control limits located at $\pm 3 \sigma$ of the average called upper and lower control limits where $\sigma$ is the standard deviation [2],[4]. The process is in control if it fluctuates within these two limits and out of control if it exceeds the limits. Process variability is usually evaluated by both the mean and variance of the quality characteristic being measured and data shown on the control chart reveal the overall direction of the production process in the future.

\section{PATTERN RECOGNITION OF CONTROL CHARTS}

Control charts can be used to detect whether they possess specific unusual patterns in order to eliminate the causes of these patterns. There are six different patterns that a quality chart may have which are [10]: normal. upward shifi. downward shift. upward trend, downward trend and cyclic as shown in Figure 1. These patterns reflect the long term behavior of the production process. In fact, they can indicate where out of control situations are likely to occur in the future. Therefore, it is useful to apply capable techniques in order identify control chart patterns to obtain information necessary to monitor the process.

There are several pattern recognition and classification techniques proposed for the recognition of patterns on control charts. Al-Ghanim and Ludeman [11] used correlation analysis to develop an automated pattern recognition tool for detecting unnatural patterns on control charts. Smith [12] implemented a backpropagation neural network to detect a shift in the mean and variance on the X-bar and R-charts. Hwarng and Ilubele [13] developed an $X$-bar control chart pattern recognizer using the backpropagation neural networks. Pham and Ortemel $|14|$ applied the learning vector quantization (LVQ) to recognize different patterns in quality charts. Guh and Hsieh [15] used artificial neural networks to recognize different patterns on quality charts and to provide estimates of pattern parameters. All these studies proved that pattern recognition techniques based on the concepts of artificial intelligence are very cffective in this area.

Neuro-fury systems have been documented as good alternatives for pattern recognition and classification. These capabilities can be put to use for 
the interpretation of patterns on control charts. The goal of using neuro-fuzzy classification is to allow the system to learn from the data collected from the production process to detect unnatural patterns. Through learning of varying patterns within control charts, the neuro-fuzzy system can decide on any pattern when faced with new inputs. In this case, the long term behavior of the production process and possible deviations can be identified.

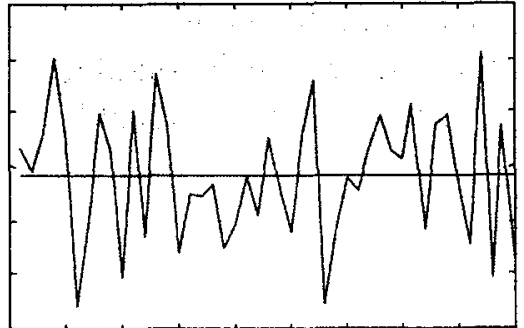

(1) Normal Pattern

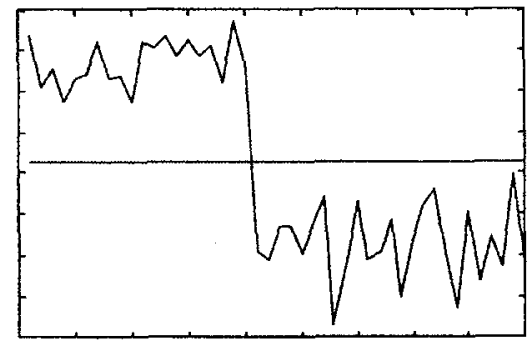

(3) Downward Shift Pattern

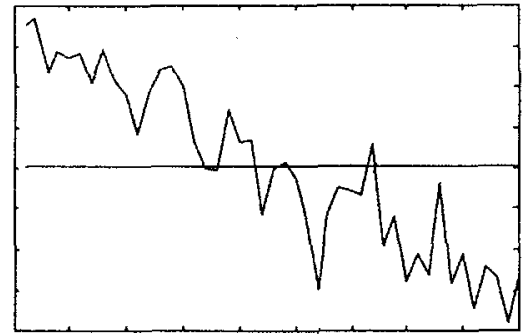

(5) Downward Trend Pattern

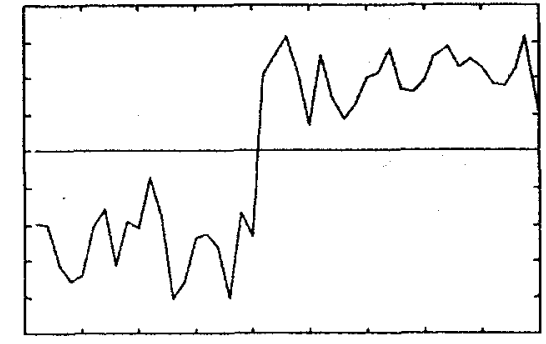

(2) Upward Shift Pattern

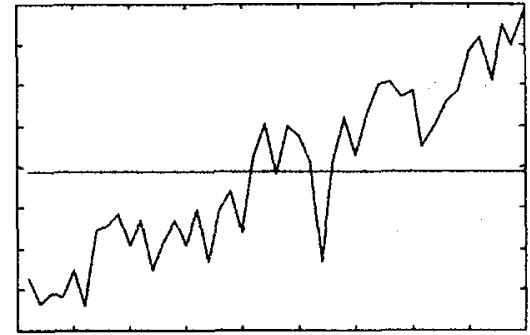

(4) Upward Trend Pattern

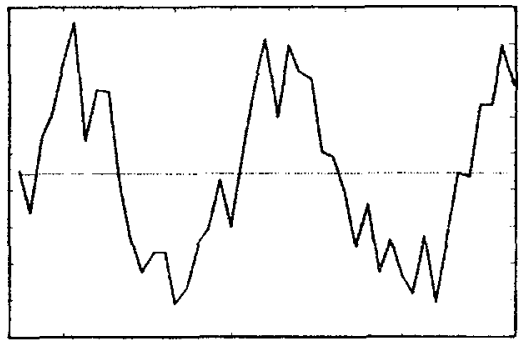

(6) Cyclic Pattern

Figure 1: Basic patterns of control charts

\section{THE NEURO-FUZZY CLASSIFICATION (NEFCLASS)}

Fvery intelligent system has some capabilities and limitations that make it suitable for particular problems and not for others. These limitations can be overcome by building a hybrid inteligent system. $A$ hybrid system consists of two or more intelligent techniques combined in a manner that overcomes the limitations of individual techniques $|5| .|16|$. 
The development of a fuzzy system requires the specification of appropriate membership functions and a set of fuzzy rules. Finding appropriate membership functions and representative fuzzy rules is a difficult task [5],[17]. Therefore, applying a learning method to a fuzzy system in order to induce the fuzzy rules from a training sample was used by many researchers to improve and hasten the development of a fuzzy system. Artificial neural networks were prime candidates for a combination with fuzzy inference systems in the form of a hybrid system because of their learning capabilities [5].

The neuro-fuzzy classification (NEFCLASS), which was originally proposed and developed by Nauck and his colleagues [9],[18],,[19], is an example of such hybrid systems where it embodies the capabilities of artificial neural networks and fuzzy inference systems. It is modeled in the form of a multilayer neural network whose weights are represented by fuzzy sets. The parameters of the fuzzy sets are modified during the learning process to achieve the best classification results where the classification task is represented in the form of fuzzy IF-TIILN rules.

Let $\Omega:\left\{c_{1}, \ldots, c_{n n}\right\}$ be a set of classes and let $x=\left[x_{1} \ldots, x_{n}\right]^{T} \in R^{n}$ be a vector and each component of $x$ expresses the value of feature. A classifier is a mapping:

$$
D: R_{n} \rightarrow \Omega
$$

A neuro-fuzzy system for classification consists of a number of fuzzy rules that can recognize the classes of unclassified inputs. The neuro-fuzzy classification (NEFCLASS) is used to derive fuzzy classification rules from a set of input data [9]. The fuzzy rules take the following form:

If $x_{1}$ is $\mu_{1}$ and $x_{2}$ is $\mu_{2} \quad \ldots$ and $x_{n}$ is $\mu_{n}$ then the pattern $\left(x_{1}, x_{2}, \ldots, x_{n}\right)$ belongs to class $i$.

where $\mu_{1} . \mu_{2}, \ldots . \mu_{n}$ are linguistic labels (i.c., high, medium, low) modeled by membership functions. The goal of NEFCLASS system is to induce these fuzzy rules from a set of data during the training process by modifying the parameters of the membership functions associated with the inputs as to correctly classify any given input patterns.

\subsection{ARCHITECTURE OF NEFCLASS}

The architecture of NEFCLASS is similar to the architecture of a feedforward neural network which consists of a huge number of interconnected units. Processing is performed within each unit using values of the incoming inputs and the weights associated with the connections between different units. The NEFCLASS system consists of three layers: input. hidden and output layer. The first layer $l$ consists of $n$ input nodes $x_{1}$. . . $x_{n}$ representing input patterns. The hidden layer is $/ I$ consists of $k \leq k_{\text {max }}$ 
nodes $R_{l} \ldots \ldots, R_{k}$ rule nodes where each node represents a fuzzy rule. The third layer is the output layer $O$ with $m$ nodes $c_{l}, \ldots, c_{m}$ representing the classes [9],[18].

The activation level $\alpha_{x}$ of an input layer node $x \in I$ depends on its external inputs. The activation level of a rule node $R \in H$ given an input pattern $p$ is [9]:

$\alpha_{R}^{\prime \prime}=\min _{r \in I}\left\{W(x, R)\left(\alpha_{x}^{p}\right)\right\}$

The activation level of an output node $c \in O$ given an input pattern $p$ is:

$\alpha_{i}^{\prime \prime}=\sum_{R \in I l} \frac{W(R, c) \alpha_{R}^{p}}{W(R, c)}$

or alternatively,

$a_{t}^{\prime \prime}=\max _{R: H}\left\{\alpha_{R}^{\prime \prime}\right\}$

where $W(x, R)$ is the fuzzy weight on the connection from input node $x$ to rule node $R . W(R, c)$ is the fuzzy weight from rule node $R$ to output node $c$ and they are equal 1 . Some of the connections coming from the same input node to rule nodes may share the same fuzzy weight. Figure 2 shows a NEFCLASS model with two input nodes, five rule nodes and two output nodes [9],[18].

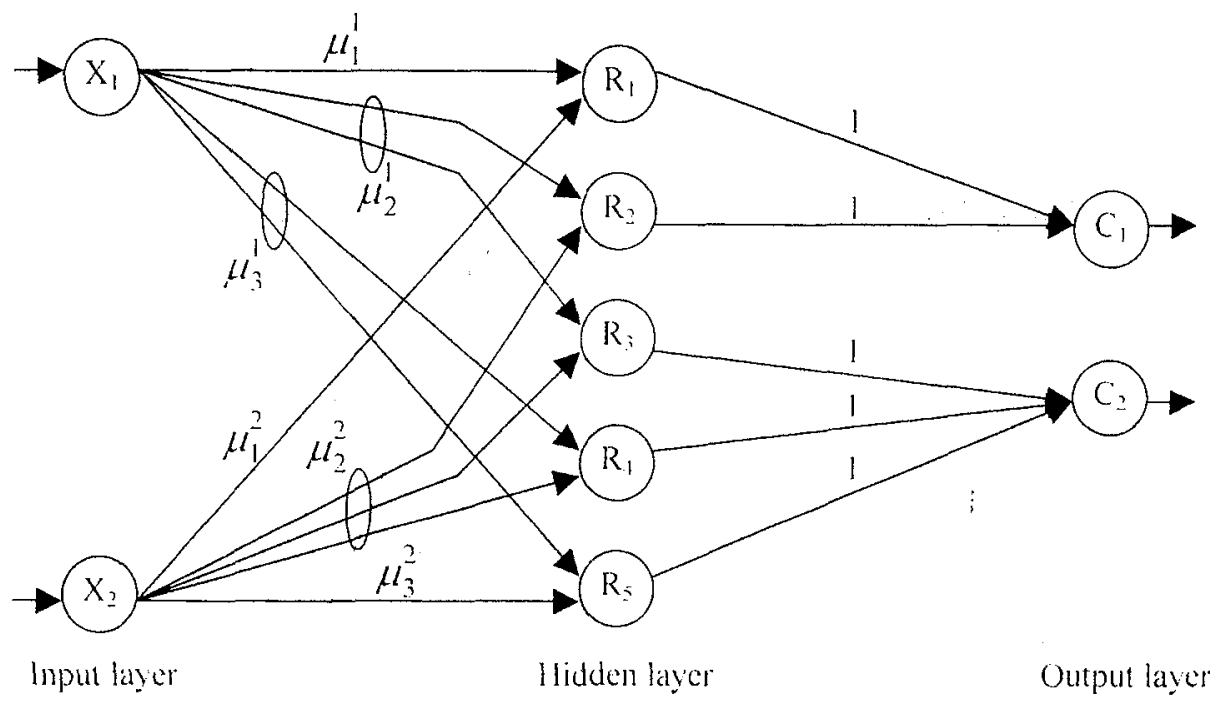

Figure 2: A NEFCLASS architecture 


\subsection{LEARNING IN NEFCLASS}

Learning in NEFCLASS is based on supervised learning where it learns using a learning procedure called fuzzy error backpropagation. The learning is performed by computing the errors at the output units of the network and propagating these errors backward through the network to adjust the parameters of the membership functions associated with linguistic terms used to represent the input variables [9]. Before learning starts, the membership functions for each input node have to be specified in addition to the maximum number of rule nodes that may be created in the hidden layer during the learning process. NEFCLASS uses the triangular membership functions to represent inputs to the system which take the following form:

$$
\mu(x)= \begin{cases}(x-c) /(a-c), & c \leq x \leq a \\ (x-b) /(a-b), & a \leq x \leq b \\ 0 & \text { otherwise }\end{cases}
$$

with $c \leq a \leq b$. The parameter $a$ gives the maximal membership grade where $\mu(x)=l$. The parameters $b$ and $c$ are the upper and lower bounds of the area and they express the amount of fuzziness in the data.

For each input $x_{i}, i=1, \ldots, n$ there are $q_{i}$ fuzzy sets $\mu_{1}^{\prime} \ldots \ldots \mu_{q i}^{\prime}$ used to represent this input. Given a set of patterns $\left\{\left(p_{l}, l_{1}\right) \ldots, \ldots\left(p_{s}, l_{s}\right)\right\}$ of $s$ patterns each consisting of an input pattern $p$ and a target pattern $t$, the learning process starts with the rule learning algorithm to create $k$ rule nodes in the rule layer. After a total of $k$ fuzzy rules are created using the training patterns where $k$ is specified by the user, the fuzzy set learning algorithm starts to adjust the parameters of the membership functions to achieve the best possible classification results [9],[18],[19].

The idea of learning in NEFCLASS is to create a fuzzy rule base first and then to modify it by adjusting the parameters of the membership functions. The fuzzy rule base is constructed by finding for each pattern in the training data a fuzzy rule that classifies it to the correct class and if a fuzzy rule with the same antecedent is not in the rule base. it will be added to the rule base [18].[19]. After the construction of the fuzzy rule base is completed. the performance of each rule is evaluated and the best rule set of rules are selected based on the number of fuzzy rules that should be stored in the rule base as specilied by the user. Then. an iterative training process is carried out to adjust the parameters of membership functions using the errors computed at the output layer in order to achieve the best classification.

\section{NEFCLASS TRAINING}

The specification of good fuzzy rules depends heavily on the training sample used to train the system. Simulation approach was used to generate 
various abnormal pattern sets. For simulation purposes, control charts were generated by the pattern generators developed in the literature in this area [11],[15] to simulate the operation of a real production process. These generators consist of a set of mathematical equations whereby each equation produces a sequence of data points that resembles the desired data set. Formulas used for generating the data for the six patterns are listed below.

Normal patterns:

$$
x(1)=n(1) \text { where } n(t)-N(0, \sigma) .
$$

Upward and downward shift patterns:

$$
x(t)=n(t) \pm u^{*} s
$$

Increasing and decreasing trend patterns:

$$
x(t)=n(t) \pm \alpha \sigma t
$$

Cyclic patterns:

$$
x(t)=n(t)+\sin (2 \pi t / \Omega) k \sigma
$$

where $t$ is the time period $(1<1<t 5), x(t)$ is the sample value at time $t, n(t)$ is the value with normal distribution $(0, \sigma)$ at time $t$ where $\sigma=2, u$ is a parameter to determine the position of shifting (before shifting $u=0$, after shifting $u=1$ ), $s$ is the shift magnitude $(s=6), \alpha$ is the slope of the trend in term of $\sigma(\alpha=1.5)$, $\Omega$ is the period of the cycle $(\Omega=20)$ and $k$ is the amplitude of the cycle in term of $\sigma(k=3)$.

Two pattern samples were generated: a training sample and a test sample. The training sample which consists of 480 patterns (80 patterns for each class), was used to train the NEFCLASS system. The test sample which has 240 patterns (40 patterns for each class). was not used in the training process but it was processed by the system after it finished training to evaluate the performance of the system. Once the input which contains 45 consecutive points representing one particular pattern processed by the system, the output will be classified into one of six predetermined categories namely normal. increasing trend. decreasing trend. upward shift. downward shift and cycle (sce Table 1).

The proposed approach was implemented using NEFCLASS software package 19].1181. Designing the NEFCLASS system involves several issues that include: 1) Deciding on the number of input. rule and output units in the network. 2) Determining the aggregation function for the output layer. 3) Selecting class names, 4) Determining the number of fuzzy sets for each input unit. Figure 3 shows the specifications of the NEFCL ASS system for the proposed approach. The network has 45 input units which is the length of each pattern. There are 50 units in the rule layer and 6 units in the output 
layer which is the number of classes. Each input variable is represented by three triangular fuzzy membership functions.

Table 1: Representation of output categories

\begin{tabular}{|lclllll|}
\hline Pattern & Outputs & & & & & \\
\cline { 2 - 6 } & 1 & 2 & 3 & 4 & 5 & 6 \\
\hline Normal & 1 & 0 & 0 & 0 & 0 & 0 \\
Down shift & 0 & 1 & 0 & 0 & 0 & 0 \\
Up Shift & 0 & 0 & 1 & 0 & 0 & 0 \\
Inc. pattern & 0 & 0 & 0 & 1 & 0 & 0 \\
Dec. pattern & 0 & 0 & 0 & 0 & 1 & 0 \\
Cyclic & 0 & 0 & 0 & 0 & 0 & 1 \\
\hline
\end{tabular}

Training the NEFCLASS system requires the specification of several training parameters that include number of training epochs and stop criteria. Training the system involves two main steps. The first is to create a total of $k$ fuzzy rules using the training pattern sample. The second step is to use the difference between the outputs of the system and actual outputs to adjust the parameters of membership functions associated with nodes in the input layer as to correctly classify input patterns.

\section{TRAINING RESULTS}

The classification accuracy level of the NEFCLASS system achieved when classifying patterns was evaluated for the training and test pattern samples. The accuracy level was calculated using the following equation:

\section{Number of patterns}

Accuracy level $=\frac{\text { correctlyclassified }}{\text { Total number of patterns tested }}$

The NEFCLASS was trained for a total of 50 epochs. Figure 4 shows the results of the training process where only 5 patterns out of 480 training patterns are misclassified. After training, the system was used to recognize patterns in the test sample where 7 patterns out of 240 patterns are misclassificd. Table 2 summarizes the classification results for the system.

Table 2: Classification results

\begin{tabular}{|c|c|c|c|c|}
\hline Sample & Classified patterns & $\begin{array}{c}\text { Misclassified } \\
\text { patterns }\end{array}$ & Total & $\begin{array}{c}\text { Accuracy } \\
\text { level }\end{array}$ \\
\hline Training & 475 & 5 & 480 & $98.95 \%$ \\
Test & 233 & 7 & 240 & $97.08 \%$ \\
\hline
\end{tabular}


During the training process, the learning algorithm induces classification rules from the training sample. The classification problem is represented in the form of fuzzy IF-THEN rules. The proposed system has 50 fuzzy classification rules where each rule is represented by one unit in the rule layer of the NEFCLASS architecture. The first rule (R_1) takes the following form (see Figure 5):

IF V 1 (input_1) is SMALL and V_2 (input_2) is SMALL AND V 3 (input 3) is MEDIUM and V 4 (input 4) is MEDIUM AND V_5 (input_5) is SMALL and V_6 (input_6) is SMALL

AND V 44 (input_44) is SMAL.L and V_45 (input_45) is SMALL THEN pattern belongs to class_5 (Decreasing Trend).

Uncertainty in the inputs was modeled using linguistic terms and each linguistic term was represented using a triangular membership function. The classification rules are obtained by modifying the parameters of these membership functions and, consequently, the shapes of the membership functions will be changed in order to derive the best classification rules. Figure 6 and 7 show membership functions for the first four input units used in the proposed system at the beginning and end of the training process.

The neuro-fuzzy approach successfully classified the majority of control charts for both the training and test pattern samples. Neuro-fuzzy systems apply the learning algorithms used to train artificial neural networks in order to achieve the lowest classification error possible. In addition. they can accommodate linguistic terms and represent the classification task in the form of fuzry rules which makes it easy to include existing body of knowledge in the classification model.

In addition to its simple learning. a NEFCLASS can be built from partial knowledge about the problem and can be refined by learning using a set of training patterns or it can be built form a set of training patterns only [9].[19]. Hence, it allows to incorporate available knowledge about the problem and to include knowledge acquired by learning. The results of the study are consistent with the idea that neuro-fuzzy approaches are good candidates for modeling complex classification problems. Their strength lies in their ability to learn very fast and generalize the results obtained from the training sample to any other sample. 


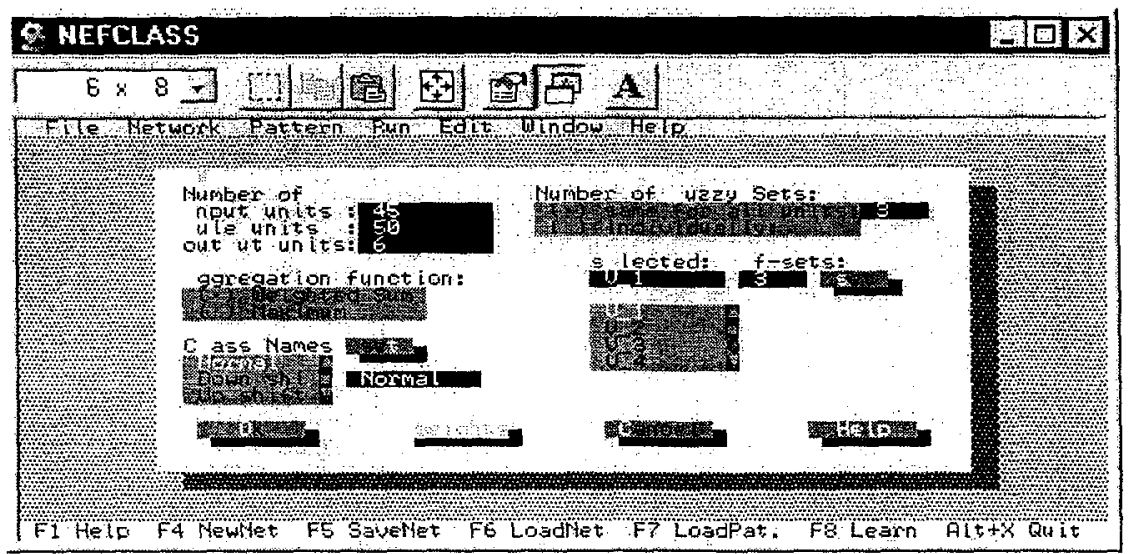

Figure 3: Design of NEFCLASS System

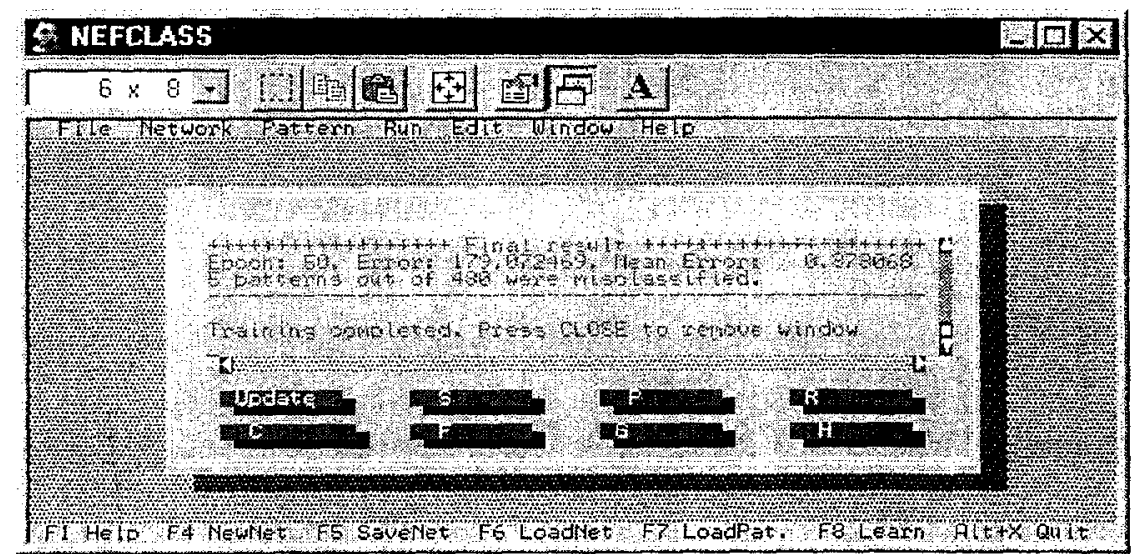

Figure 4: Training Results of NEFCLASS

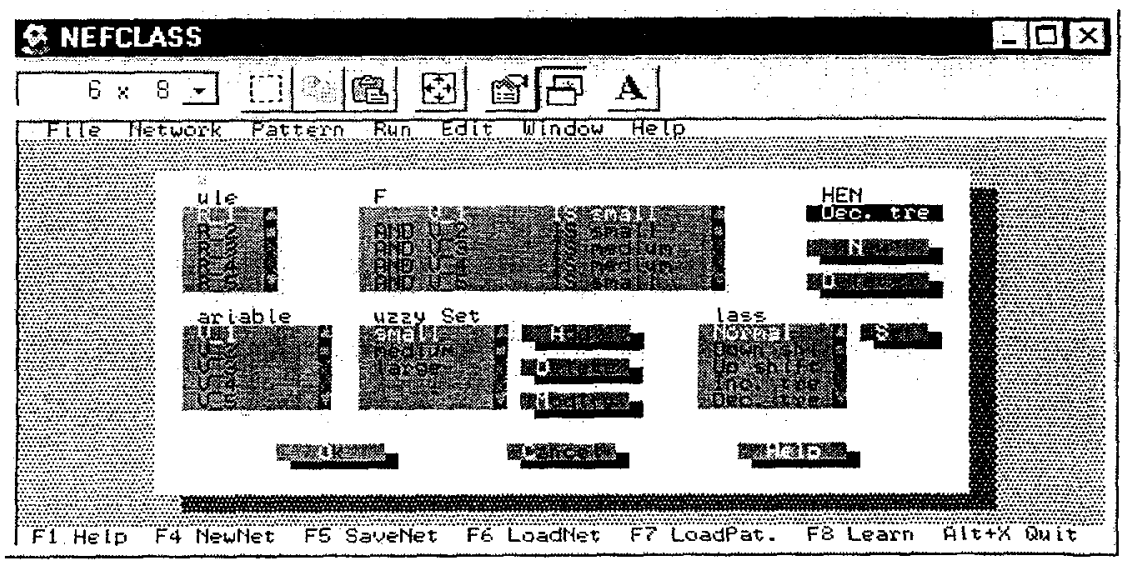

Figure 5: The First Fuzzy Rule of NEFCLASS (R_1) 


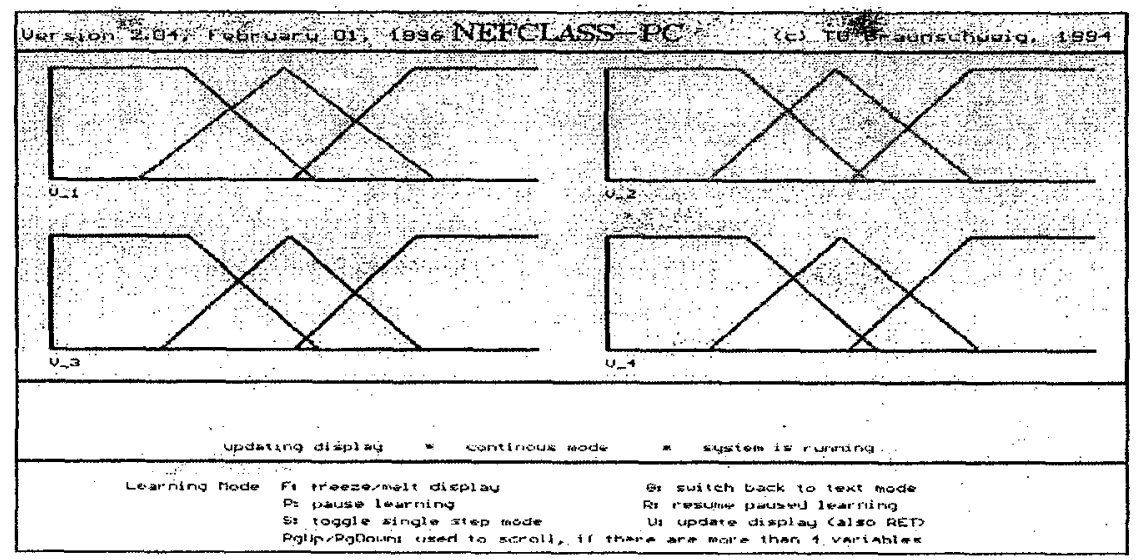

Figure 6: Membership Functions at Beginning of Training

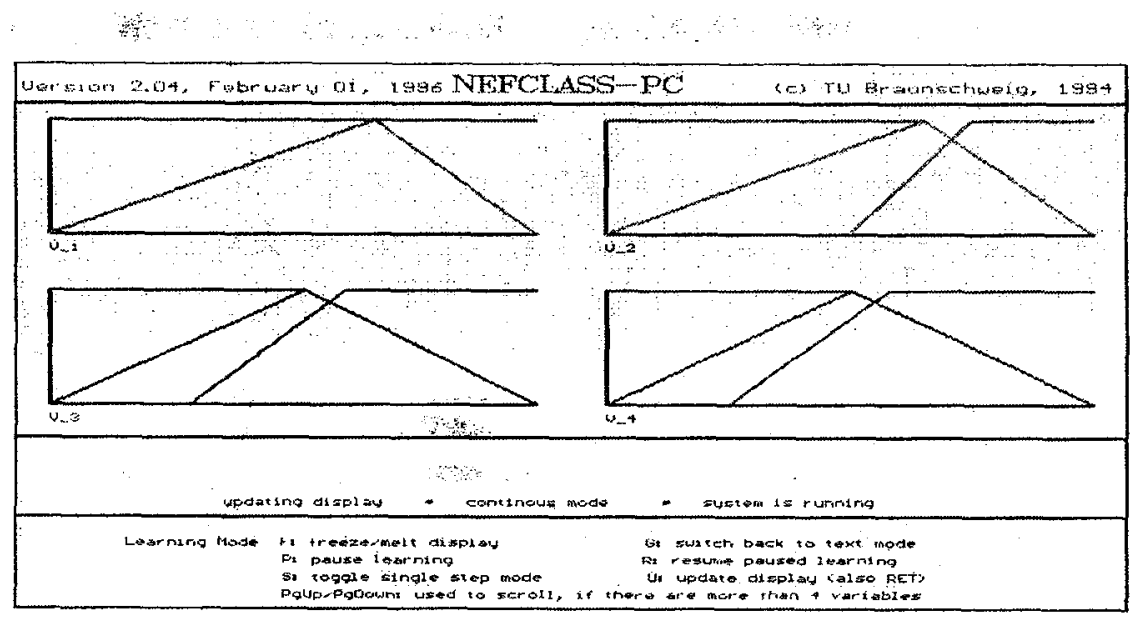

Figure 7: Membership Functions at End of Training

$$
\text { ats }
$$




\section{CONCLUSION}

Quality is a major factor that impacts the ability of an organization to sustain competition and succeed in its business. Organizations spend considerable efforts to ensure that their products are produced with high quality standards. Control charts are primary tools used in quality control to determine whether the production process is within control or not in order to take corrective actions if the process is out of control. In addition, patterns shown on a quality chart reveal important information about the behavior of the process in the future. Identifying such patterns can reveal important information about abnormal behavior of the process where unacceptable variations may occur.

Neuro-fuzzy systems are hybrid systems combining the capabilities of artificial neural networks and fuzzy inference systems. This paper presented a neuro-fuzzy classification system for identifying different patterns on control charts. The proposed system was trained using a training pattern sample that was randomly generated using pattern generators. After training was completed. the system was tested using a test sample in order to test its performance. The neuro-fuzzy classification system showed outstanding performance in identifying different patterns on quality charts for both the training and test pattern samples. Advantages of the neuro-fuzzy systems compared to other techniques include their ability to learn very fast from a set of training data and to represent the problem in the form of fuzzy rules that are easy to interpret.

\section{REFERENCES}

[1] Delworth, James B. Operations Management Design, Planning and Control for Manufacturing and Services. New York: McGraw Hill, Inc., 1992.

[2] Evans. James R., and William M. Lindsay: The Management and Control of Quclity, St. Paul, MN USA, West Publishing Company, 1993.

[3] Chase, Richard B. and Aquilano, Nicholas J. Production and Operations management: A Life Cycle Approach, Homewood, IL: Irwin, Inc., 1992.

141 Oakland. John S. Statistical Process Conirol. Oxford: Heinemann, 1996.

151 Nauck. D. "Beyond neuro-fuzzy: perspectives and directions." Proceedings of the Third European C'ongress on Intelligent Techniques and Sofi Computing (EUFIT'95), Aachen. August 1995, pp. 1159-1164.

16] Nauck. D. "Neuro-fuzzy systems: review and prospects." Proceedings of the Fifih European Congress on Intelligent Techniques and Sofi ('ompuling (EUFIT'97). Aachen. September 1997. pp. 1044-1053.

17| Jang. Jyh-Shing Roger. (1993): " 1 NIFIS: Adaptive-Network-Based Fur Inference System." IEEE Transactions on Sistems, Man and Cybernetics. Vol. 23, No. 3, pp. 665-685.

181 Nauck, D. and Kruse, R.: "NEFCON-I: An x-window based simulator for neural fuzay Controllers." Proceedings of the IEEE International conference on Neural Netrorks. Orlando, 1995. pp. 1638-1643. 
[9] Nauck, D. and Kruse, R.: "NEFCLASS- a neuro-fuzzy approach for the classification of data." Proceedings of the ACM Symposium on Applied Computing, Nashville, ACM Press, February 1995, pp. 461-465.

[10] Grant, E.L. and Leavenworth, R.S. Statistical Process Control. New York, NY: McGraw-Hill Book Company, 1988.

[11] Al-Ghanim, Amjad M. and Ludeman, Lonnie C. (1997): "Automated unnatural pattern recognition on control charts using correlation analysis techniques." Computer \& Industrial Engineering, Vol. 32, pp. 679-690.

[12] Smith, A.E. (1994): "X-bar and R control chart interpretation using neural computing." International journal of Production Research, Vol. 32, pp. 309-320.

[13] Hwarng, H.B. and Hubele, N.F. (1993): "Backpropagation pattern recognizers for X-bar control charts: methodology and performance." Computer and Industrial Engineering, Vol. 24, pp. 219-235.

[14] Pham, D.T. and Oztemel, E. (1994): "Control chart pattern recognition using learning vector quantization networks." International Journal of Production Research, Vol. 32. No. 3, pp. 721-729.

115| Guh. Ruey-Shy and Hseih. Yi-Chih. (1999):"A neural network based model for abnormal pattern recognition of control charts." ('omputer \& Industrial Engineering, Vol. 36, pp. 97-108.

[16] Jang, Jyh-Shing Roger and Sun, Chuen-Tsai : "Neuro-fuzzy modeling and control." The Proceedings of the IEEE, March 1995, Vol. 83, No. 3, pp. 378-406.

[17] Jang, J.-S. R.; Sun C.-T. and Mizutani. E. Neuro-Fuzzy and Soft Computing: A Computational Approach to Learning and Machine Intelligence. USA: Prentice Hall, Inc., 1997.

[18] Nauck, D. and Kruse, R. (1997): "A neuro-fuzzy method to learn fuzzy classification rules from data." Fuzzy Sets and Systems, Vol. 89, pp. 277-288.

[19] Nauck. D.; Nauck, U. and Kruse, R.: "Generating classification rules with the furzy system NEFCLASS." Proceedings of Biennial Conference of the North American Fuzzy Information Processing Sociely (NAFIPS 96), Berkeley. CA. June 1996. pp. 466-470. 


\title{
نظام تصنيف عصبي فازي لتمييز أثماط خرائط الرقابة على الجودة
}

\author{
د. مندي عبدالله الهندي \\ أستاذ مشارك - قدم الأساليب الكمية

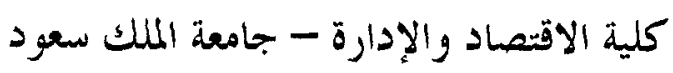

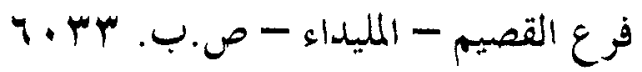

ملخص: لاقت النظم العصبية الفازية في السنو ات الماضية إهتماما متز ايدا وبأأت تستخلاد

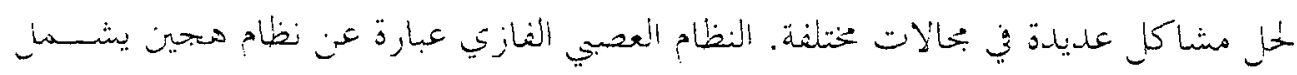

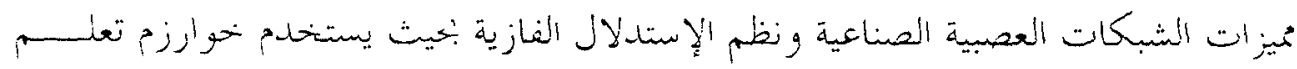

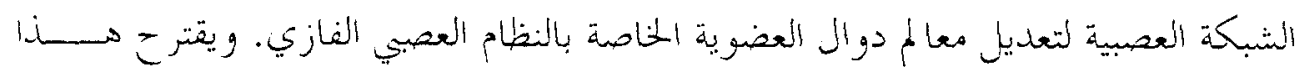

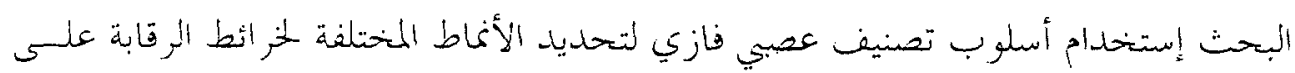

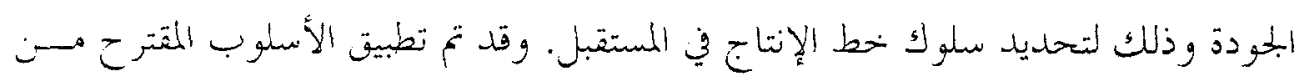

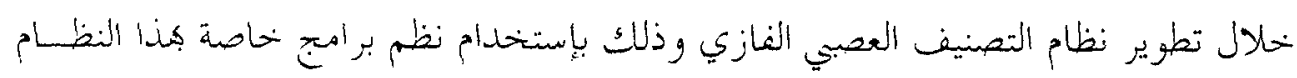

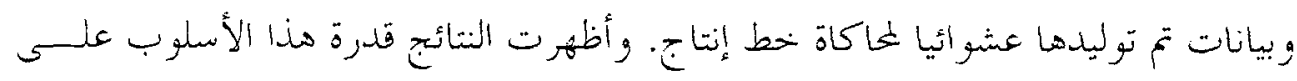

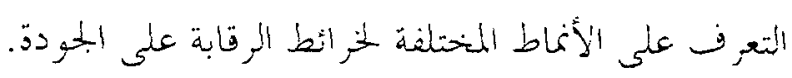

